\title{
MOLECULAR RYDBERG SPECTROSCOPY MAGNETIC FIELD EFFECTS IN ALKYL HALIDES
}

\author{
S.P. YcGlynn, J.D. Scott* and W.S. Felps \\ Chemistry Department, The Louisiana State University, Bâton Rouge, LA 70803, \\ U.S.A. \\ ${ }^{*}$ Chemistry Department, The University of Montona, Missoula, MT 59812, U.S.A.
}

\begin{abstract}
Résumé. - Le moment angulaire total de tous les niveaux du premier complexe $s$ de

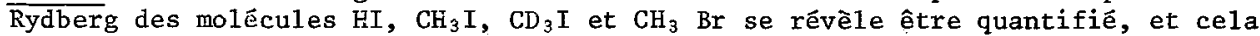
même dans les molécules de symétries $C_{3}$ pour lesquelles 1 a brisure de symétrie implique 1'absence d'une telle quantification. Ceci suggère que la symétrie effective est celle d'un "bit" moléculaire dans lequel la transition est localisée tandis que le reste de la molécuie se comporte de manière perturbatoire. Les moments angulaires des différents états vibroniques ont aussi été mesurës. On a trouvé que des vibrations non totallement symétriques couplées à la partie électronique produisent souvent des changements importants du moment magnétique. Une approche perturbationnelle complětement molëculaire permet d'expliquer toutes les observations. Elle comporte des termes décrivant (i) la déviation de la molécule de la non-linéarité;(ii) les effets Renner-Teller;(iii) les effets Jahn-Teller.
\end{abstract}

Abstract. - The total angular momentum of all states of $\mathrm{HI}, \mathrm{CH}_{3} \mathrm{I}, \mathrm{CD}_{3} \mathrm{I}$ and $\mathrm{CH}_{3} \mathrm{Br}$ in the 1st s-Rydberg complex is found to be quantized, even in $C_{3}$, molecules where symmetry-breaking decrees the absence of such quantization. Tisis observation suggests that the operative symmetry is that of a molecular "bit" within which the transition is localized and that the remainder of the molecule is merely perturbatory. The angular momenta of various vibronic states have also been measured. It is found that non-cotally symetric vibrations coupled to electronic origins of ten produce profound changes of magnetic moment. A perturbation approach of "whole molecule" nature is developed and is capable of explaining all observations. The perturbation expression includes terms descriptive of (i) deviation of the molecule from non-linearity; (ii) Renner-Teller effects; and (iii) Jahn-Teller effects.

1. Introduction. - There exists no reason why the phenomenology associated with the Rydberg states of molecules should be less formidable or less interesting than that for atoms. Indeed, the molecular realm should be considerably richer. It is worth emphasizing, therefore, that the diatomic molecule, while a good starting point for molecular excursions, is not a truly representative "molecule". Indeed, the diatomic molecule cannot exhibit (i), vibronic effects, such as Renner-Teller or Jahn-Teller [1] phenomena; (ii), optical activity of molecular nature; (iii), stability, when the molecule is loaded with more vibrational energy than the minimum required for its dissociation; (iv), interactions of discrete-continum nature (i.e., auto-dissociations) which branch into more than one dissociative channe1; (v), anti-resonances caused by interactions of a discrete state with a bound-bound intravalence transition unless, for some reason, the density of states in the bound-bound continum is abnormally high; and (vi), a total symmetry-breaking of angular momentum quantization. For these reasons, this work will emphasize nonlinear polyatomic molecules.

Zeeman measurements on polyatomic molecules are not usually feasible: Molecular band widths are normally larger than the splitting produced by accessible cryogenic magnet field strengths. Consequently, resort to other experimental Zeeman variants is necessary. The variant used in this work is magnetic circular dichroism (MCD) $[2,3]$. The experimental technique, particularly in the VUV region, is a difficult one which requires the whole panoply of constant-intensity high-flux VUV sources; $\mathrm{CaF}_{2}$ optics; $\mathrm{MgF}_{2}$ photoelastic modulators; high-field ( $\left.7 \mathrm{~T}\right)$ 
end-corrected, long-path length $(\sim 10 \mathrm{~cm})$ cyrogenic magnets; and detectivity adequate to the task of extracting information from a background which may be 3 to 5 orders more intense. Nonetheless, the MCD technique is the method of choice because of the ease of analysis of the data and the fact that all the detail inherent in conventional polarized Zeeman spectroscopy is retained.

The MCD measurement is defined as the difference of absorptivity of left-handed $(1 \mathrm{cp},-)$ and right-handed circularly polarized (rcp, +$)$ light of a particular frequency $\nu_{0}$ in a field-on situation. That is

$$
\Delta A^{H}\left(\bar{v}_{0}\right) \equiv A_{-}^{H}\left(\bar{\nu}_{0}\right)-A_{+}^{H}\left(\bar{\nu}_{0}\right)
$$

where $\mathrm{H}$ denotes magnetic field strength. The corresponding theoretical expression $[2,4]$ when the ground state is non-degenerate and certain small approximations are imposed is

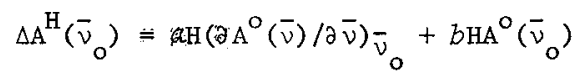

where the quantity a carries all the information on the paramagnetism of the excited state and $b$, the diamagnetic component, describes field-induced state mixings. Furthermore, it is expected that $\underline{b} \ll a$. The advantage of Eq. 2 is best utilized by simultaneous generation of $A^{\mathrm{o}}(\bar{v}), \partial \mathrm{A}^{\mathrm{O}}(\bar{v}) / \partial \bar{v}$ and $\Delta \mathrm{A}^{\mathrm{A}}(\bar{v})$ versus $\bar{v}$ curves. A "following" of the $\Delta \mathrm{A}^{\mathrm{H}}(\bar{\nu})$ and $\partial \mathrm{A}^{\mathrm{O}}(\bar{v}) / \partial \bar{v}$ plots indicates that the excited state at $\bar{v}$ is paramagnetic and that it possesses a magnetic moment proportional to the ratio of curve ordinates. A following of $\Delta \mathrm{A}^{\mathrm{H}}(\bar{\nu})$ and $\mathrm{A}^{\mathrm{O}}(\bar{\nu})$, on the other hand, indicates diamagnetism of the excited state at $\bar{v}$.

\section{EXPERTMENTAL RESULTS}

The results for the lst $\mathrm{s}$-Rydberg complex of $\mathrm{CH}_{3} \mathrm{I}$, methyl iodide, are shown in Figure 1. This configurational excitation, $5 p \rightarrow 6 s$ of the iodine component, yields four excited states which; in order of increasing energy, are denoted [5] ${ }_{\Pi_{2}},{ }^{3} \Pi_{1}$, ${ }^{3} \Pi_{0}$ and ${ }^{1} \Pi_{1}$. If the ground state, ${ }^{1}{ }_{\Sigma}{ }^{+}$be labeled 0 ; then the excited states may be labeled $1,2,3$ and 4 , again in order of increasing excitation energy. The corresponding transitions $(0 \rightarrow 1,0 \rightarrow 2$, etc.) are simply labeled $1,2,3$ and 4 in Figure 1.

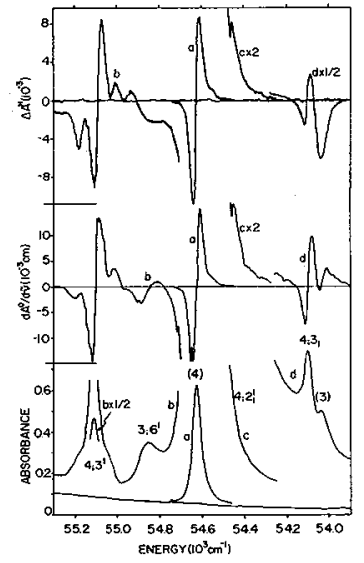

Fig. 1a: Absorption, derivative, and MCD spectra of methyl iodide.

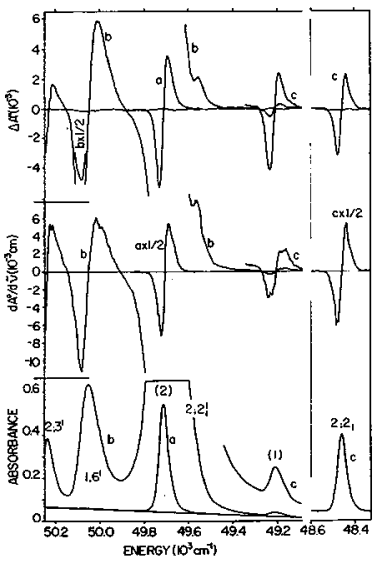

Fig. 1b: Absorption, derivative, and vicD spectra of methyl iodide. 
Origin 3 , which is occluded by a hot-band $4 ; 3$ in $\mathrm{CH}_{3} \mathrm{I}$, becomes distinct and unobscured in $\mathrm{CD}_{3} \mathrm{I}$ because of a large decrease of the vibrational intensity of $v_{3}$ in the per-deutero compound. The measured magnetic moments (in Bohr magnetons) are given in the following tabulation, where they are compared to that expected on the basis of simple $(\Lambda, \Sigma)$ - coupling.

EXPERIMENT

\begin{tabular}{|c|c|c|c|c|c|}
\hline STATE & $\underline{\mathrm{HI}}$ & $\mathrm{CH}_{3} \mathrm{I}$ & $\mathrm{CD}_{3} \mathrm{I}$ & $\mathrm{CH}_{3} \mathrm{Br}$ & $(\Lambda, \Sigma)$ \\
\hline 4 & & 1.3 & & 1 & $I$ \\
\hline 3 & & 0 & 0 & & 0 \\
\hline 2 & 1.34 & 1.6 & & 1 & 1 \\
\hline 1 & 3 & 2.7 & 2.9 & & 3 \\
\hline
\end{tabular}

Results for the 1st $\mathrm{p}$ - and d-complexes of $\mathrm{CH}_{3} \mathrm{I}$ are shown in Figure 2. Certain states may be identified immediately on the basis of absorptivity, MCD signal-type and MCD $\alpha$-term signal intensity.

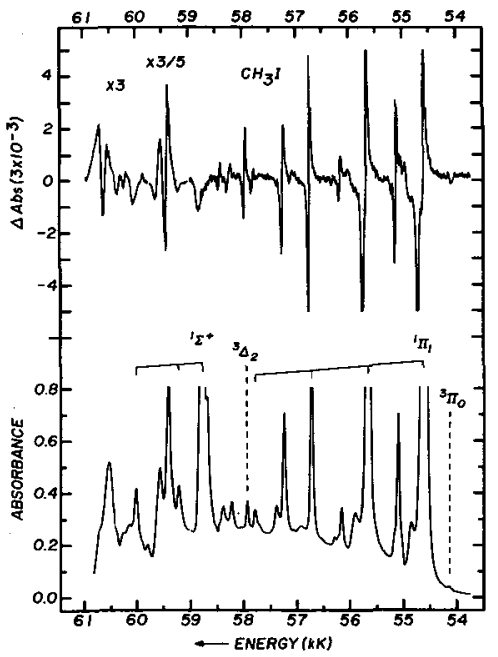

Fig. 2: Absorption and ISCD spectra of methyl iodice.

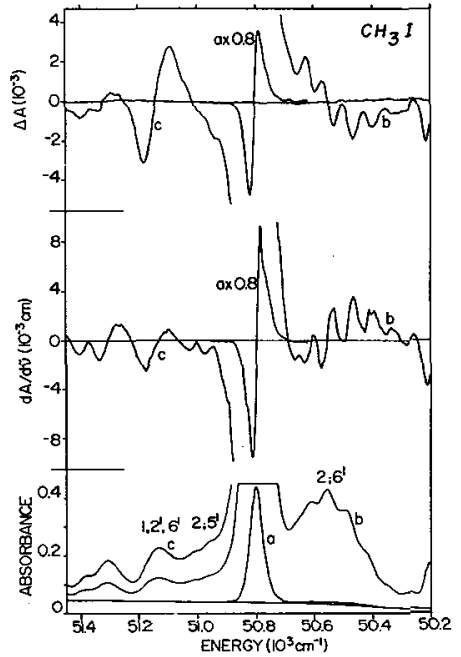

Fig. 3: Absorption, derivative, and MCD spectra of methy 1 iodide.

These states are labelled in Figure 2. Certain vibrational activities are also evident in Figure 2: A three-member progression of a totally symmetric mode built on the $\pi_{1}$ origin is readily identified, as are two single-member progressions in each of two different modes built on the ${ }^{1} \Sigma^{+}$origin. The power of the MCD technique for VUV studies of molecules is amply demonstrated in Figures 1 and 2.

We now emphasize the existence of some oddities. It is found that a nontotally symmetric vibration built on origins 2 or 4 may exhibit an inverted $a$ signal, a normal a signal, a $b$ signal or even a null signal. A clear example is given in Figure 3 , where the $2 ; 61$ band exhibits a paramagnetic characteristic of equal magnitude but opposite sign to that of the origin band for state 2 (see Figure 1). Indeed, it is now known that coupling of non-totally symmetric vibrational modes can convert an $a$-type origin into $a-,-a-, b-$ or 0 -vibronic 
states. It is with the meaning of these alterations that the remainder of this work is concerned.

\section{PRIMITIVE MODEL}

The objective is a model of the various electrostatic forces that affect electronic motion in molecular systems, a model which, simultaneously, can account qualitatively for spin-orbit coupling in the several limits. Since the ultimate purpose is to provide a context within which to discuss MCD spectra, the model must facilitate precise statements concerning magnetic phenomena.

The best zero-order starting point is the linear (triatomic) molecule in the $(\Lambda, S)$-spin-orbit coupling limit. In this limit the space and spin coordinates remain separate and simple considerations of electrostatic (spatial) perturbations can be indulged. Concurrently, one may consider other spin-orbit coupling limits in the $(\Lambda, S)$-basis, remembering that, because of the azimuthal space isotropy, the angular momentum $\Omega= \pm \Omega \hbar \underset{\sim}{\mathrm{k}}$ ( $\mathrm{k}$ being the unit vector in the internuclear axis), must be the same, in a linear molecule, for all spin-orbitally mixed states.

The most convenient spatial coordinate system is the cylindrical system: $z$, internuclear axis; $\rho$, perpendicular distance from $z ; \phi$, azimuth from an arbitrary but fixed plane containing 2 . The electronic wavefunction, expressed in these and the spin coordinates, $S$ and $\Sigma$, has the form

$$
\Psi=\psi_{0}(\bar{\rho}, \bar{z}, \bar{\phi}) \psi_{S}(S, \Sigma)
$$

where $\bar{\rho}, \bar{z}$ and $\bar{\phi}$ indicate the spatial coordinates of all electrons. The spacedependent function, $\psi_{0}$, may be expressed as

$$
\psi_{0}(\bar{\rho}, \bar{z}, \bar{\phi}) \equiv \psi_{ \pm}(\bar{\rho}, \bar{z}, \bar{\phi})=\mathrm{F}_{ \pm}(\bar{\alpha}) \mathrm{X}(\bar{\rho}, \bar{z}) \exp ( \pm i \Lambda \phi)
$$

where $\bar{\alpha}$ represents the set of phase angles between $\phi$ and the azimuth $\phi_{1}$ for each individual electron (where $\phi_{\mathrm{k}}$ refers to the $\mathrm{kth}$ electron). In other words, $\alpha_{k}=\phi_{k}-\phi$ and $\bar{\alpha}=,\left\{\alpha_{k}\right\}$. For an $n$ electron system, $\Lambda$ may be expressed in terms of the individual $\lambda_{j}^{\prime} s$ as $\Lambda=\left|\sum_{j=1}^{n} \lambda_{j}\right| ; \lambda_{j}=\lambda_{j} \cdot \Lambda_{\sim} / \hbar{ }^{2} \Lambda$.

Although $\Lambda$ is restxicted to positive values, $\lambda$ for the $j$ th individual electron may be either positive or negative according to the contribution which that electron makes to the total momentum $\underset{\sim}{\Lambda}$. The phase function, $F_{ \pm}$, is

$$
F_{ \pm}(\bar{\alpha})=\exp \left( \pm i \sum_{j=1}^{n} \lambda_{j} \sum_{k=1}^{n} \lambda_{k} \alpha_{k} / \Lambda\right)
$$

where positive and negative signs differentiate the double-degenerate components with $\Lambda \neq 0$.

In addition to the various spin-orbit coupling cases, we will now treat al1 other systems as perturbed $(\Lambda, S)$-coupled, linear triatomics. Since these latter perturbations will be electrostatic in nature, their primary effect occurs only on the spatial functions. These systems are 
(i) Non-linear molecule (loss of spatial isotropy in $\phi$ );

(ii) Vibronic coupling in a linear triatomic;

(iii) Vibronic coupling in a non-linear molecule.

However, before proceeding with the electrostatic perturbations, the general effects of spin-orbit coupling will be reviewed in the context of the zero-order model. In strong-coupling cases, where $\Lambda$ and $\sum$ lose uniqueness and $\Omega$ remains as the only "good" electronic quantum numb̌er, ex̃cited states which are electronic-dipole forbidden in the $(\Lambda, S)$-limit may gain spin-allowedness by coupling with states having $S=0$ (and $\Sigma=0$ ). The original "parent state" then gains spin-allowedness by mixing with contaminant singlet states. This terminology, of course, is meaningful only in the context of perturbation theory. The mixing occurs because the spin-orbit perturbation Hamiltonian contains terms that are totally symmetric in the double group $\mathrm{C}_{\infty}^{*} \hat{i}$. Thus, $\Omega$ of the parent state and $\Omega$ of the contaminant state $(\mathrm{s})$ must be the same. Since the basis is formed in the $(\Lambda, S)-1$ imit, it follows that

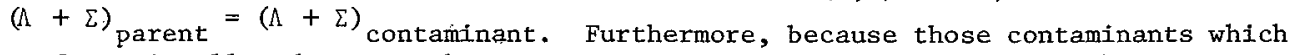
confer spin-allowedness on the parent state must have $\Sigma=0$, we find that

$\Lambda_{\text {contaminant }}=(\Lambda+\Sigma)$ parent $=\Omega_{\text {parent }}$.

Since the electrostatic perturbation (i) - (iii) are functions only of the spatial coordinates, they cannot induce spin-orbit coupling. The "parent states", then, in the electrostatic cases are the spin-orbit coupled resultants expressed in the $(\Lambda, S)$-basis.

\section{(i) Non-Linear Molecule:}

Consider the atom "C" of the linear molecule A-B-C to consist of m (finite) identical atoms symmetrically disposed about the internuclear axis. The symmetry of this system is now $C_{m}$ for $m \geq 2\left(C_{s}, m=1\right)$. The electrostatic potential associated with these $\mathrm{in}$ atoms is

$$
\hat{H}^{\prime}=\sum_{k=0}^{\infty} V_{k}(\bar{z}, \bar{p})[\exp (i k m \phi)+\exp (-i k m \phi)]
$$

where the phase angle between the nuclear (off-axis) potential and $\phi$ is set equal to zero -- a simplification that does not affect generality. The loss of isotropy in $\phi$ may affect the parent states in two ways:

--States with $\Lambda$ different from that of the parent may "admix" and destroy the integer nature of $\Lambda$ (parent).

---A degenerate parent state may split into two non-degenerate perturbed states.

We now examine these effects from the standpoint of symmetry. The effect of $\hat{H}^{\prime}$ on a degenerate pair, $\psi_{ \pm}(\bar{\varphi}, \bar{a}, \bar{\phi})$, of parent states is determined in first order by the secular determinant ${ }^{ \pm}$

$$
\left|\begin{array}{ll}
\mathrm{H}^{\prime}{ }_{+}-\mathrm{E}^{\prime} & \mathrm{H}_{+-}^{\prime} \\
\mathrm{H}^{\prime}{ }_{+} & \mathrm{H}^{\prime}{ }_{--}
\end{array}\right|=0
$$


In defining the matrix elements, the simplification is made that the parent state is a pure state in the $(\Lambda, S)$-basis. This, of course, is not true in other coupling limits. However, given a complete $(\Lambda, S)$-basis, the wavefunction in any other coupling limit can be expressed as a linear combination of the $(\Lambda, s)$-functions. Hence, the treatment in any other limit is not more complicated but merely longer. The elements of the determinant are readily expanded. It is found that the components of $\hat{\mathrm{H}}^{\prime}$ that can remove degeneracy are those for which $\mathrm{km} / 2$ is integer, and the particular states for which splitting can occur are those for which $\mathrm{A}$ is equal to one of these integers. In general, when is even, the levels for which degeneracy is removed are those with $\Lambda=(\mathrm{m} / 2) \ell$ where $\ell=1,2,3,4 \ldots$ When $\mathrm{m}$ is odd, those levels for which $\Lambda=\mathrm{m}$, lose degeneracy. For levels which remain degenerate, the perturbation energy, $\mathrm{E}^{\prime}$, is

$$
E^{\prime}=v_{0}
$$

and for those which lose degeneracy, it is

$$
E^{\prime}=v_{0} \pm v_{j} ; j=\frac{2 \Lambda}{m}=\text { integer. }
$$

The first-order energy corrections lead to degeneracy removal and provide a context (the values of $v_{k}$ ) in which to discuss these splittings. A knowledge of the perturbation wavefunctions (i.e., the nature of the contaminant states) leads to several fundamental statements: For example, the mechanism by which a forbidden state may gain allowedness. The perturbed state to first-order is

$$
\psi_{1}=\psi_{1}^{0}+\sum_{j=2}^{\infty} c_{j} \psi_{j}^{0}
$$

where the subscript $I$ represents the parent state. The values of $c_{j}$ are given by

$$
\begin{gathered}
c_{j}\left(E_{1}^{0}-E_{j}^{0}\right)=F_{ \pm, j}^{*} F_{ \pm, 1} \sum_{k=0}^{\infty} \int_{\infty}^{+\infty} \int_{0}^{\infty} x_{j}(\bar{z}, \bar{\rho}) x_{l}(\bar{z}, \bar{\rho}) \bar{\rho} V_{k}(\bar{z}, \bar{\rho}) d \bar{\rho} \bar{z} \\
\quad \underline{x} \int_{0}^{2 \pi} \exp \left(+i \Lambda{ }_{j} \phi\right)[\exp (i k m \phi)+\exp (-i k m \phi)] \exp (i \Lambda, \phi)
\end{gathered}
$$

Thus, the coefficient $\mathrm{c}_{\mathbf{j}}$ is non-zero only if

$$
\Lambda_{1}+\Lambda_{j}=-k m, k=0,1,2,3, \ldots
$$

or

$$
\Lambda_{1}-\Lambda_{j}= \pm \mathrm{km}, \mathrm{k}=0,1,2,3, \ldots
$$

If Eq. 12 applies, state $j$ mixes with its orbital angular momentum vector $\Lambda_{j}$ antiparallel to that of the parent state. Equation 13 is the condition for ${ }^{j}$ paralle1 mixing.

\section{(ii) Vibronic Coupling in a Linear Molecule (Renner-Teller)}

Perturbations arising from the coupling of vibrational and electronic motions are discussed in the context of the Franck-Condon Principle. The perturbation 
Hamiltonian is written in a form pertinent to the $(\Lambda, S)$-1inear triatomic basis. The initial discussion of vibronic coupling refers only to linear triatomics where the perturbation Hamiltonian consists, in toto, of Renner-Teller terms. Discussion of the interactions in $\mathrm{C}_{\mathrm{m}}$, systems is based on the linear-molecule results, but with the required addition of Jahn-Teller terms.

The vibrational motion necessitates inclusion of normal mode coordinates. It is because of separability of these coordinates that the Franck-Condon Principle holds. The possibility of observing vibronically-forbidden transitions requires a mixing of Franck-Condon-allowed states into the forbidden parent. This mixing must be investigated on the basis of the perturbation Hamiltonian, full use being made of the vibrational wavefunction. In a linear triatomic one of the vibrations (the degenerate $\pi$ vibration) is non-totally symmetric and is associated with Franck-Condon-forbidden states. Appropriate coordinates for this vibrational mode are given in Figure 4. The electronic coordinate $\phi$ serves as a reference for this coordinate system. The vibration itself is depicted in Figure 5 .

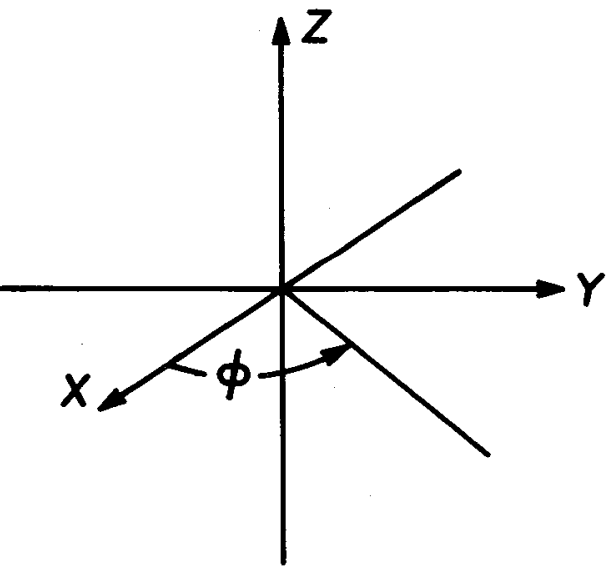

Figure 4: Relationship between nuclear coordinates, $\mathrm{x}$ and $\mathrm{y}$, and electronic coordinate $\phi$.
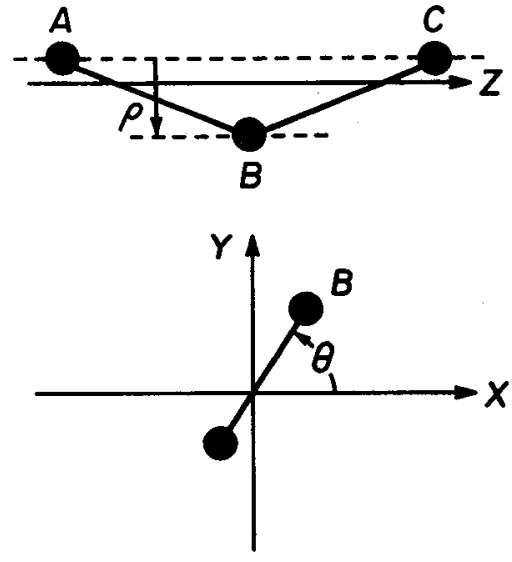

Figure 5: Geometry of $\pi$ vibrational mode of a linear triatomic molecule.

The vibrational wavefunction for such a motion is

$$
\psi_{\mathrm{n}^{\prime}, \ell, \pm} \equiv \psi_{\mathrm{v} \pm}=\mathrm{f}_{\mathrm{n}^{\prime}, \ell}(\rho) \exp ( \pm i \ell \theta)
$$

where $\rho=\left(x^{2}+y^{2}\right)^{1 / 2} ; n^{\prime}$ is the number of quanta of the excited mode; and

$$
\ell=n^{\prime}, n^{\prime}-2, \ldots, 1 \text { or } 0 \text {. }
$$

In the following discussion, the $\theta$-dependent part of Eq. 14 is of primary concern.

The vibrational part of the Hamiltonian may be written 


$$
\begin{aligned}
& \hat{H}^{\prime}=\sum_{n=0}^{\infty} z^{n} \sum_{\substack{j=0 \\
j, k}}^{\infty} \sum_{\substack{k=0 \\
j, k \text { even }}}^{\infty} a_{j k n} \rho^{j}\left(e^{i k \phi} e^{-i k \theta}+e^{-i k \phi} e^{i k \theta}\right) \\
& \left.+\sum_{\substack{j=1 \\
j, k \text { odd }}}^{\infty} \sum_{\substack{j k n \\
j}}^{\infty} a^{j}\left(e^{i k \phi} e^{-i k \theta}+e^{-i k \phi} e^{i k \theta}\right)\right\}
\end{aligned}
$$

where, because of our concerns, the primary interest lies in the $\phi$ - and $\theta$-dependent parts of Eq. 16.

For a state that is double degenerate, both electronically and vibrationally (i.e. $\ell \neq 0$ ), the product wavefunction is

$$
\psi_{\mathrm{ev}}=\psi_{ \pm} \Psi_{\mathrm{v} \pm}
$$

Since all four combinations are possible, zero-order quadruply-degenerate vibronic states arise. For convenience, these four states are labeled

$$
\psi_{1}=\psi_{+} \psi_{v_{+}} ; \psi_{2}=\psi_{+} \psi_{v_{-}} ; \psi_{3}=\psi_{-} \psi_{v^{+}} ; \text {and } \psi_{4}=\psi_{-} \psi_{v-}
$$

The first-order energy correction is obtained from

$$
\left|H^{\prime}{ }_{t u}-\delta_{t u} E^{\prime}\right|=0 ; t, u=1,2,3 \text {, or } 4
$$

The only non-zero off-diagonal elements are $\mathrm{F}_{23}^{\prime}=\mathrm{H}_{132}^{\prime} \%$ and these are non-zero only if $\ell=\Lambda$. In this case, the degeneracy of states 2 and 3 is removed, and the zeroorder parent states become the appropriate symmetric $\left(\Sigma^{+}\right)$and antisymmetric $\left(\Sigma^{-}\right)$ linear combinations of functions 2 and 3. Thus, the first-order energy effect is essentially the splitting of degeneracy of states 2 and 3 . States 1 and 4 cannot mix and remain degenerate. For cases where $\Lambda \neq l$, mixing cannot occur, states 2 and 3 remain degenerate, and second-order energy corrections are required in order to remove the degeneracy of the pair $\psi_{1}, \psi_{4}$ or the pair $\psi_{2}, \psi_{3}$.

Information more germane to the present concern can also be derived from first-order effects. The question devolves on whether or not $\hat{H}^{\prime}$ will permit a forbidden parent state to $\mathrm{mix}$ with an allowed contaminant state and, if it does, how, in terms of their angular momenta, these states admix. Since the term "allowed" is defined in the context of the spin, orbital and vibrational components of the ground state and since the ground state is always taken to be vibrationally unexcited, the allowed excited states in the $(A, S)-$, non-vibronically-interacting basis must satisfy

$$
\Lambda=0, \pm 1 ; S=0 ; \Sigma=0 \text {; and } \ell=0 \text {. }
$$

Consideration of Eq's. 16 and 17 leads to the following conclusions: 
---The parent states 1 and 4 cannot mix in the first-order of $\mathrm{H}^{\prime}$ with any state that is both orbitally and Franck-Condon allowed.

---For the insertion of first-order allowedness, it is required that the parent state have

$$
\Lambda-2=0, \pm 1
$$

---For the cases, $\Lambda-\ell= \pm 1$, degeneracy remains. In the event that $\Lambda-\ell=$ +1 , the parent and contaminant mix with parallel $\Lambda$ vectors, while for $\Lambda-\ell=-1$ the mixing is antiparallel. This is an important feature because of the sensitivity of MCD to such mutual angular momentum directionalities.

(iii) Non Linear, $\mathrm{C}, \mathrm{m}>3$, molecules (Jahn-Teller Effect). It is assumed that degenerate vibrations in $\mathrm{C}_{\mathrm{m} v}$ molecules can be discussed in terms of Eq. 14 with the coordinate $\theta$ now defined as

$$
\begin{aligned}
\theta & \equiv \cos ^{-1}\left[\xi_{a} /\left(\xi_{a}^{2}+\xi_{b}^{2}\right)^{1 / 2}\right] \\
& \equiv \sin ^{-1}\left[\xi_{b} /\left(\xi_{a}^{2}+\xi_{b}^{2}\right)^{1 / 2}\right]
\end{aligned}
$$

where $\xi_{a}$ and $\xi_{b}$ are the normal coordinates of the degenerate pair of vibrations.

The perturbation Hamiltonian in the $\mathrm{C}_{\mathrm{m}}$, system is now

$$
\begin{aligned}
& H^{\prime}=\sum_{\alpha=0}^{\infty} z^{\alpha} \sum_{j=0} \sum_{k=0} a_{j k \alpha} \rho^{j}\left(e^{i k \phi} e^{-i k \theta}+e^{-i k \phi} e^{-i k \theta}\right) \\
& +\sum_{j=0} \sum_{k=0} \sum_{n=1} a_{j k \alpha n} \rho^{j}\left[\left(e^{i(k+n m) \phi} e^{-i k \theta}+e^{-i(k+n m) \phi} e^{i k \theta}\right)\right. \\
& \left.\left.+\left(e^{i(k-n m) \phi} e^{i k \theta}+e^{-i(k-n m) \phi} e^{i l i \theta}\right)\right]\right\}
\end{aligned}
$$

Equation 20 contains all the elements of Eq. 16 (ire, the Renner-Teller terms) plus a set of other terms which evolve from the non-linear symetry (the Jahn-Teller terms). Utilization of Eq. 20 permits expansion in terms of the linear-molecule basis. Those of the Jahn-Teller terms that contain $\mathrm{m}$ in the exponential account for the off-axis perturbations directly attributable to non-linearity.

The more complex Hamiltonian of Eq. 20 multiplies vibronic-coupling possibilities relative to the linear triatomic case. The following observations may be made:

- - States 1 and 4 (cf. Eq. 17) lose degeneracy whenever $\ell+\Lambda=\mathrm{nm}$, $\mathrm{n}=1,2,3, \ldots$ Also, states $I$ and 4 , even while retaining degeneracy, can now mix, in first-order, with totally-allowed states.

---States 2 and 3 lose degeneracy when

$$
\Lambda-\ell=0, \pm n m ; n=1,2,3, \ldots
$$

---The rules for the mixing of allowed contaminants are

$$
\Lambda-\ell=0, \pm 1,
$$


which arise from the Renner-Teller effects, and

$$
\Lambda \pm \ell= \pm 1+n m ; n=1,2,3, \ldots
$$

where, in the latter case, all possible sign combinations are permitted.

The perturbation Hamiltonian of Eq. 20 also accounts for the $\mathrm{C}_{\mathrm{m}}$ perturbation in the zero-vibronic-interaction limit. Thus, an electronic state miv a $\mathrm{C}$ molecule may be described by the quantum numbers of the "ancestor" state $\mathrm{m}$. the linear basis. The vibrational quantum numbers $n^{\prime}$ and $\ell$ also retain their linearmolecule identities.

\section{DISCUSSION}

In using the various coupling conditions developed under items (i), (ii) \& (iii), some presumptions must be borne in mind. The first of these is that motions which make a paramagnetic contribution some $10^{-3}$ times smaller than the electronic contribution, do not, of themselves, contribute to the observed magnitude of $a$-terms. The second is tisat the magnetic moment of an excited state is predominantly that of the "parent" state whereas the intensity may well be totally attributable to that of a "contaminant" state. In situations where this latter is the case, the phasing of $a$-terms, positive or negative, is a direct consequence of the phasing of parent/contaminant angular momenta. With these comments in mind, it is a relatively simple matter to conclude that an a-type parent can yield, as a result of mixing, an $\alpha$-type, - $a$-type, or $b$-type resultant (where $|b|$ may be zero) whereas a $b$-type parent will normally yield only a $b$-type resultant.

We now illustrate the alteration of $\alpha$-signatures using origin 2 and vibronic states built on 2 .

Transition 2 to the ${ }^{3} \pi_{1}$ state possesses a large oscillator strength. It is clearly electric-dipole allowed. It exhibits paramagnetism and the magnetic moment is approximately $1_{\mu_{B}}$. Mixing of ${ }^{3} \Pi_{1}$ with $1_{\Pi_{1}}$ is permitted by the spin-orbit coupling operator, but only in such a way that the two angular momenta remain paralle1. Thus, the MCD signature of 2 should be in phase with that of state 4 (i.e., the ${ }^{1} \mathrm{I}$ state) and both should exhibit comparable $a$-magnitudes. Such is, in fact, the case.

The $2 ; 5^{1}$ and $2 ; 6^{1}$ transitions exhibit $a$-terms comparable to that of state 2 but of opposite signature. The $2: 4^{1}$ transition exhibits either a null term or a very weak $b$-term. All those absorption bands, while considerably less intense than transition 2, are relatively strong. Since all bands should be Franck-Condonforbidden, vibronic coupling must account for the observed intensity. ${ }_{1}^{\text {Now }}$, RepnerTeller terms remove the degeneracy of state 2 and 3 (Eq. 17) to yield $\Sigma^{+}$and $\Sigma$ states neither of which will exhibit a magnetic moment and only one of which, namely ${ }_{1 \Sigma}{ }^{+}$, is permitted to connect via dipole radiation to the ground state. Thus, the $2 ; 4$ transition must occur as a result of Renner-Teller coupling. Jahn-Teller terms, on the other hand, mix Franck-Condon-allowed $\Pi_{l}$ states(states $1 \& 4$ of Eq. 17) but only in such a way that the angular momenta of parent and contaminant are antiparallel. Thus, a negative $a$-term should resilt. Since these conclusions are congruent with observations for the $2 ; 5^{2}$ and $2 ; 6^{1}$ states, we must conclude that these states obtain intensity as a result of Jahn-Teller mixing.

It is assumed that these few examples illustrate the detailed information which can be extracted from Rydberg spectra using the MCD technique.

\section{CONCLUSION}

MCD techniques provide a nice means of measuring magnetic moments, assigning vibrational progressions in the totally symnetric modes, detecting vibronic bands which depnd on $\mathrm{J}-\mathrm{T}$ or R-T effects for their intensity, and determining the operative route, $\mathrm{J}-\mathrm{T}$ or $\mathrm{R}-\mathrm{T}$, which produces that intensity. 


\section{ACKNOWLEDGEMENT}

This work was supported by the U.S. Department of Energy. The advice of Professor Gary L. Findley (New York University) is deeply appreciated.

\section{REFERENCES}

[I] HERZBERG, G., Molecular Spectra and Molecular Structure. III. Electronic Spectra and Electronic Structure of Polyatomic Molecules (Van Nostrand Reinhold Co.) 1966.

[2] SCOTT, J.D., FELPS, W.S., FINDLEY, G.L. and McGLYNN, S.P., J. Chem. Phys. 68 (1978) 4678 .

[3] SCOTT, J.D., FELPS, W.S. and McGLYNN, S.P., Nuclear Instruments and Methods 152 (1978) 231 .

[4] Stephens, P.J., Adv. Chem. Phys. 35 (1976) 197.

[5] FELPS, W.S., HOCHMANN, P., BRINT, P. and McGLYNN, S.P., J. Mol. Spectrosc. 59 (1976) 355. 